\title{
Tratamiento de las fracturas de cuello femoral con osteosíntesis. Diez claves para el éxito
}

\author{
Fernando M. Bidolegui, Sebastián Pereira, Gabriel Vindver \\ Servicio de Ortopedia y Traumatología, Hospital Sirio Libanés, ECICARO, Ciudad Autónoma de Buenos Aires, Argentina
}

\begin{abstract}
RESUMEN
Las fracturas de cuello femoral son un cuadro habitual para el cirujano traumatológico y cuando se elige realizar una osteosíntesis, son muchos los factores por considerar para conseguir un buen resultado y evitar las complicaciones. En este artículo de actualización, intentamos dar 10 claves para el éxito cuando se opta por la reducción y la osteosíntesis como método terapéutico. Palabras clave: Fractura; cuello; fémur; osteosíntesis.
\end{abstract}

Treatment of Femoral Neck Fractures with Osteosynthesis. Ten Keys to Success

\section{ABSTRACT}

Femoral neck fracture is a pathology that the trauma surgeon usually faces. When he or she decides to perform osteosynthesis, there are many factors to consider in order to achieve a good result and avoid complications. In this update article we seek to offer 10 keys to success when reduction and osteosynthesis are chosen as the therapeutic method.

Key words: Fractures; neck; femur; osteosynthesis.

\section{INTRODUCCIÓN}

La fractura del cuello femoral es un cuadro habitual al que se enfrenta el traumatólogo y su tratamiento constituye aún un verdadero desafío. Tiene una presentación bimodal, afecta, en gran medida, a pacientes añosos por traumatismos de baja energía, pero también a un pico menor y creciente de pacientes jóvenes que sufren traumatismos de alta energía. Cuando se elige realizar una osteosíntesis, son muchos los factores por considerar para obtener un buen resultado y evitar las complicaciones que, en algunos reportes, llegan hasta el $45 \%{ }^{1,2}$ Sobre la base de nuestra experiencia y una prolija revisión bibliográfica, intentaremos revisar los aspectos técnicos de la osteosíntesis de la fractura de cuello femoral para reducir, al mínimo, los errores, así como identificar modificadores de la evolución, para reconocer grupos de riesgo con osteosíntesis y, de esa manera, poder hacer una mejor indicación terapéutica y, si se elige la osteosíntesis, optimizar sus resultados.

\section{OBJETIVOS}

Intentamos resumir, en 10 tópicos, distintos aspectos para obtener los mejores resultados cuando se decide realizar una osteosíntesis en una fractura de cuello femoral.

\section{Momento adecuado para la cirugía}

Cuánto afecta la evolución de una fractura de cuello femoral, i.e., el tiempo transcurrido desde el trauma hasta la osteosíntesis es un tema controversial, clásicamente se sugiere la operación antes de las 6 h para disminuir la incidencia de necrosis. 
Hace tres décadas, Manninger y cols. ${ }^{1}$ compararon las cirugías realizadas antes de las $6 \mathrm{~h}$, entre las $6 \mathrm{y} 24 \mathrm{~h}$ y luego de las $24 \mathrm{~h}$, y comunicaron que la tasa de seudoartrosis y necrosis fue significativamente inferior en el primer grupo.

Sin embargo, en 2009, Loizou y cols. ${ }^{2}$ evaluaron a 1023 pacientes con fracturas mediales de cadera tratados con osteosíntesis y tiempos de espera entre el trauma y la cirugía que oscilaron entre $<6$ h y 48 h. No hallaron diferencias significativas en la incidencia de necrosis.

Upadhyay y cols. ${ }^{3}$ compararon dos grupos de pacientes con fractura de cuello femoral desplazadas que fueron sometidos a reducción y osteosíntesis. La incidencia global de necrosis fue del 16,3\%, y no hallaron diferencias significativas entre los pacientes operados antes de las $48 \mathrm{~h}$ o después.

En 2015, Papakostidis y cols. ${ }^{4}$ realizaron un metanálisis que evaluó el intervalo anterior a la fijación interna de las fracturas de cuello femoral. Estos autores concluyeron en que faltan estudios bien diseñados y que, si bien la cirugía debe realizarse tan rápido como sea posible, no hay evidencias claras respecto a en qué medida la reducción del tiempo hasta la cirugía afecta la evolución de la fractura, pero sugieren que, después de las 24 h, podría aumentar la tasa de seudoartrosis.

En nuestra serie de 93 pacientes con fracturas mediales tratadas con fijación interna, el tiempo promedio de espera entre la fractura y la cirugía fue de 4.6 días, con una tasa de necrosis del 5,3\% que no difiere de la comunicada en la mayoría de las series y, si bien tratamos de operar lo antes posible, una espera $>6 \mathrm{~h}$ o $24 \mathrm{~h}$, habitual en nuestro medio, no modifica nuestra indicación. ${ }^{5}$

\section{Adecuada clasificación}

Clasificar las fracturas de cuello femoral es muy importante y se debe distinguir entre desplazadas y no desplazadas. Las primeras incluyen las Garden tipos I y II; y las desplazadas, las Garden tipos III y IV. Sin embargo, esto, muchas veces, es dificultoso, porque la cadera está en rotación externa y acortada, lo que torna difícil interpretar las imágenes radiográficas.

En 2008, Egol y cols. ${ }^{6}$ comunican que las radiografías con tracción y rotación interna ayudan a mejorar sensiblemente la capacidad de clasificar las radiografías respecto al trazo y el desplazamiento, y tienen un impacto directo en la decisión terapéutica.

Respecto a la proyección necesaria para la evaluación inicial de esta fractura, habitualmente, se sugiere la incidencia de Lowenstein; sin embargo, al darle rotación a la cadera, se corre el riesgo de desplazar más la fractura y de dañar más la vascularización. Por lo tanto, es recomendable realizar un perfil quirúrgico y, de esta forma, no movilizar la cadera lesionada.?

Definir el grado de desplazamiento tiene una relación directa con los resultados si la elección es la osteosíntesis. Frandsen y cols. ${ }^{8}$ publicaron que los pacientes con fracturas de cuello femoral no desplazadas tuvieron una tasa de complicaciones del 5\% (necrosis o seudoartrosis), en tanto que la tasa fue del $40 \%$ en aquellos con fracturas desplazadas, sin diferencia estadísticamente significativa.

En su serie de 93 casos, Pereira y cols. ${ }^{5}$ informan una tasa de complicaciones del 4,2\% en pacientes con fracturas no desplazadas, claramente inferior al $36,3 \%$ de aquellos con fracturas desplazadas.

Por lo tanto, las radiografías con tracción y rotación interna representan una herramienta simple, efectiva y económica para poder determinar, con mayor exactitud, el trazo fracturario y también el desplazamiento (Figura 1).

\section{Evaluación de la conminución posterior}

El grado de conminución es un factor predictivo de una tasa más alta de complicaciones o de malos resultados cuando se trata una fractura de cuello femoral con reducción y fijación interna. Huang y cols. ${ }^{9}$ evaluaron a 146 pacientes y dividieron los resultados según si había conminución posterior o no, con un seguimiento de 4.6 años. Comprobaron, con significancia estadística, que los pacientes con conminución posterior tuvieron un mayor acortamiento, una tasa de necrosis más alta y más cantidad de conversiones a artroplastia.

Rawall y cols. ${ }^{10}$ observaron una tasa de seudoartrosis significativamente más alta en pacientes con fracturas cervicales tratados con osteosíntesis cuando tenían conminución posterior.

Por último, Pereira y cols. ${ }^{5}$ informaron la consolidación sin necrosis en 10 de 14 casos $(71,4 \%)$ sin conminución y en 4 de 8 casos $(50 \%)$ con conminución.

Por lo expuesto, consideramos que, cuando se planea una osteosíntesis en pacientes con fracturas de cuello femoral, es necesario contar no solo con un adecuado estudio radiográfico, sino también con una tomografía computarizada (TC) que nos permita evaluar la cara posterior del cuello femoral, sabiendo que si hay conminución aumenta significativamente la posibilidad de falla con este método terapéutico. 


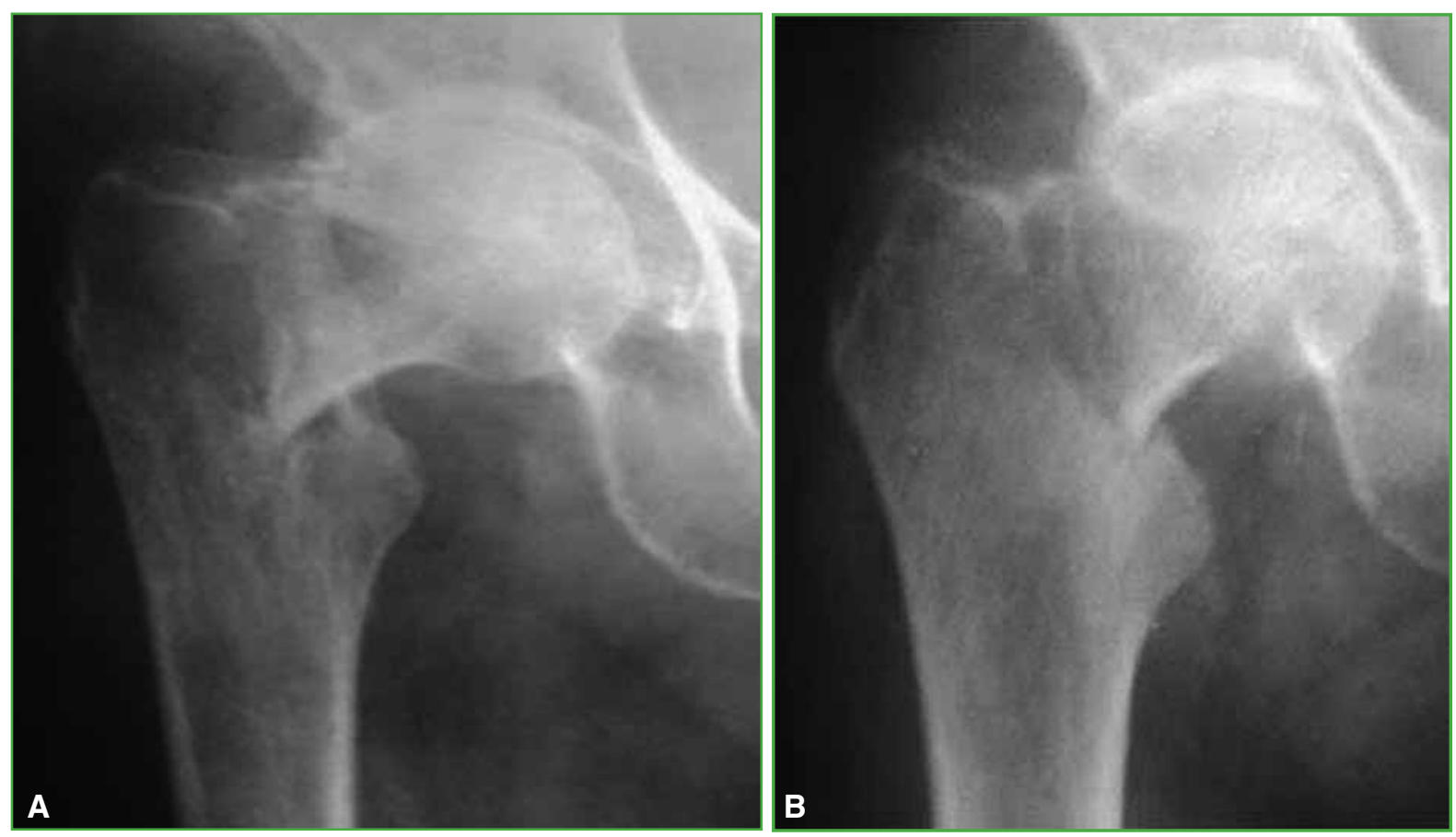

Figura 1. A. Radiografía de cadera, de frente, sin tracción, donde se dificulta la visualización del trazo fracturario. B. Al realizar tracción y rotación interna, se despliega el cuello y se identifica un trazo medial desplazado.

La conminución posterior del cuello femoral podrá valorarse con la radiografía de perfil quirúrgico, en los cortes axiales de la TC o la resonancia magnética (RM). En todas, se manifiesta con una imagen de desplazamiento posterior del segmento cervicocefálico (Figura 2).
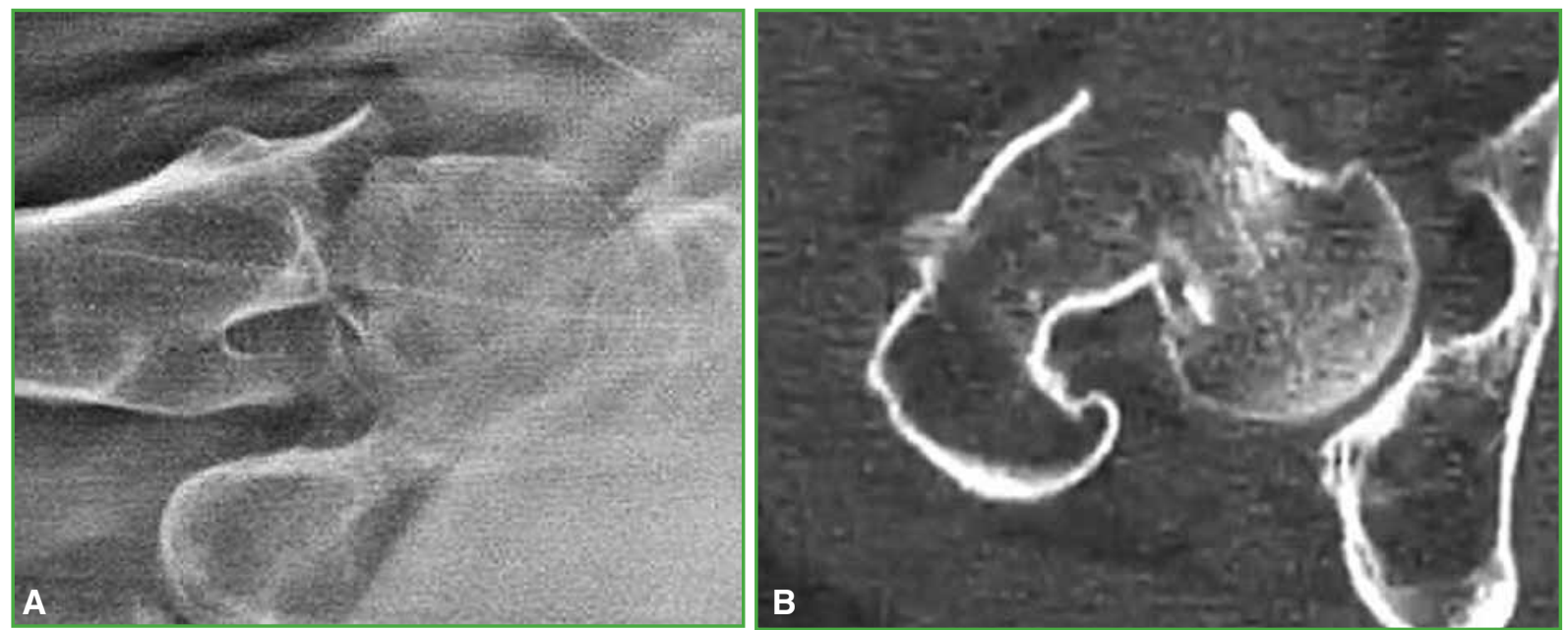

Figura 2. Paciente con fractura de cuello femoral. A. Radiografía de perfil quirúrgico. Se observa la conminución.

B. Tomografía computarizada. Se aprecia la conminución posterior. 


\section{Posición del paciente}

La posición del paciente, como así también el uso de la mesa de tracción o mesa común dependen de la preferencia del cirujano. Algunos prefieren colocar al paciente en decúbito dorsal con un realce debajo del glúteo, en una mesa común radiolúcida. En general, esto requiere un asistente para mantener la tracción y manipular la extremidad durante el procedimiento. Si bien el uso de este tipo de mesas requiere de más ayudantes y más destreza por parte del radiólogo, una de sus ventajas es tener toda la extremidad libre y la posibilidad de realizar maniobras de reducción cerrada con más comodidad.

La mesa de tracción tiene la gran ventaja de poder controlar y mantener la reducción, y permite la visualización con el equipo de rayos sin mayores inconvenientes. Existen diferentes formas de colocar al paciente en ella. La posición de "tijera" coloca la cadera no lesionada extendida y en eje, pero en un plano más inferior, y la cadera fracturada en extensión para permitir la fluoroscopia lateral. Otra opción es flexionar la cadera no lesionada y colocarla en una "piernera de obstetricia" y, por último, la colocación con la pierna no fracturada (¿sana?) en abducción que permite la entrada del intensificador de imágenes entre las piernas (Figura 2).

\section{Adecuada visualización}

Para una correcta reducción y osteosíntesis de una fractura de cuello femoral, es necesaria una correcta y completa visualización no solo del cuello y la cabeza femoral, sino también de la diáfisis tanto de frente como de perfil.

Rikli y cols. ${ }^{11}$ señalan que es evidente la falta de entrenamiento para obtener y evaluar imágenes del fémur proximal, de perfil. Poyanli y cols. ${ }^{12}$ presentan un método para poder mejorar y simplificar la imagen del fémur proximal en el perfil, compensando la anteversión del fémur y, por lo tanto, dejando una imagen que es paralela al piso y que contribuiría a evaluar, con más facilidad, la reducción y la colocación del implante.

Por este motivo, se deben hacer todos los esfuerzos posibles para poder observar bajo intensificador de imágenes, en el frente y el perfil, la articulación coxofemoral, el cuello, la zona trocantérica y la diáfisis femoral, antes de colocar los campos quirúrgicos e iniciar el procedimiento.

Una vez logradas las imágenes deseadas, resulta útil poner una marca en el piso para señalar el lugar donde deberá volver a ubicarse el arco en "C" luego de colocar los campos quirúrgicos (Figura 3).

\section{Reducción}

La reducción junto con el desplazamiento inicial es uno de los factores predictivos más importantes del éxito de la cirugía de osteosíntesis en fracturas de cuello femoral. La reducción anatómica es obligatoria y se deberá intentar conseguirla por todos los medios.

Yang y cols. ${ }^{13}$ analizan 202 fracturas de cuello femoral en pacientes con una edad promedio de 64 años e informan que la posibilidad de seudoartrosis es 2,93 veces (OR) más alta en aquellos con fracturas desplazadas que no desplazadas, mientras que el riesgo aumenta 18,92 veces si la reducción de la fractura fue deficiente.

Florschutz y cols. ${ }^{14}$ evaluaron 51 fracturas cervicales desplazadas en pacientes jóvenes (15-50 años) y en cuatro de los cinco $(75 \%)$ pacientes con una reducción no anatómica, se produjeron complicaciones (necrosis o seudoartrosis).

Chua y cols. ${ }^{15}$ evaluaron a 108 pacientes con fractura desplazada de cuello femoral e identificaron a la reducción en varo como un factor determinante de falla en el $31 \%$ de los casos, y demostraron que este defecto es estadísticamente significativo como factor predictivo de mal resultado.

En su artículo de actualización sobre el tratamiento de las fracturas de cuello femoral, Florschutz y cols. ${ }^{14}$ confirman que el ángulo en varo, la caída posterior, la brecha y los defectos de rotación son factores que aumentan significativamente la incidencia de seudoartrosis, pérdida de la reducción y osteonecrosis.

Por último, Pereira y cols. ${ }^{5}$ demuestran una relación estrecha entre la calidad de la reducción y la tasa de complicaciones, y hacen hincapié en la necesidad de recurrir a una reducción abierta y fijación interna si no se consigue una reducción anatómica a cielo cerrado. Obtuvieron una tasa de consolidación del 88,9\% en las fracturas desplazadas tratadas con reducción abierta y fijación interna y del $46 \%$ en aquellas con reducción cerrada $(\mathrm{p}>0,05)$.

Por lo tanto, es imperativo lograr una reducción anatómica. No deberá aceptarse ningún varo, valgo $>15^{\circ}$ ni desejes en el perfil $>15^{\circ}$. Si se decide una reducción y osteosíntesis de una fractura de cuello femoral, esta reducción se debe efectuar a cielo cerrado o abierto. Y no deben aceptarse reducciones subóptimas por el hecho de evitar una cirugía abierta. 


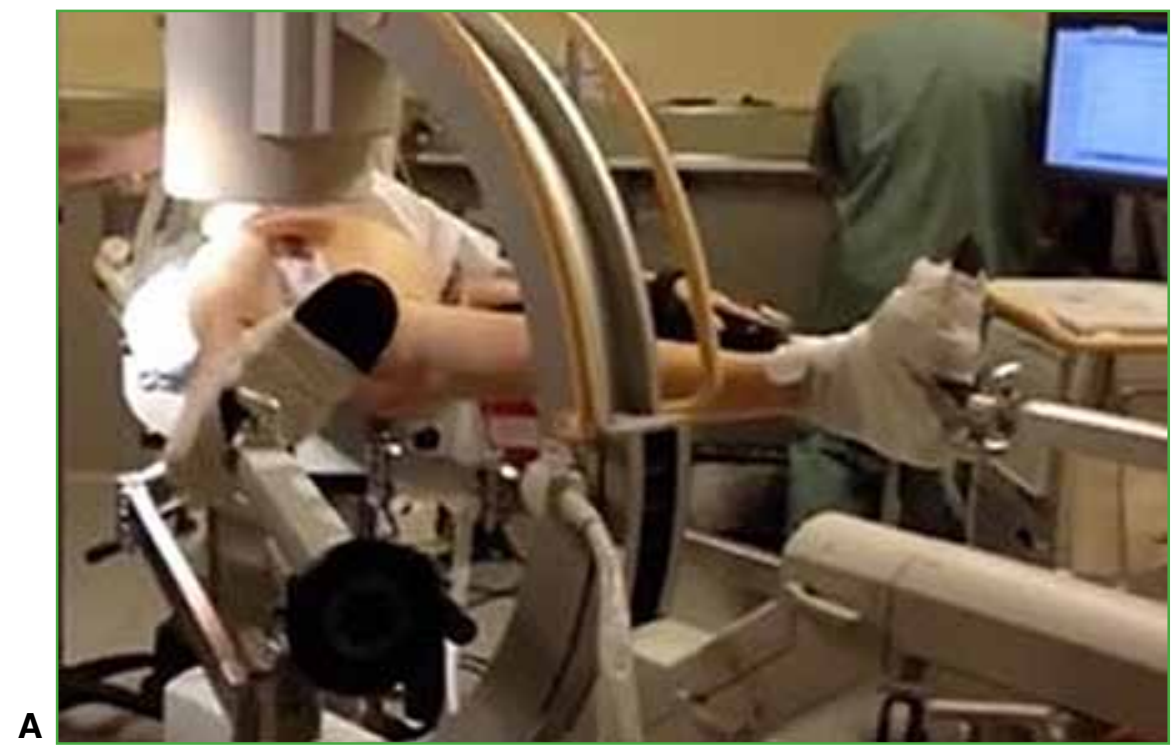

A
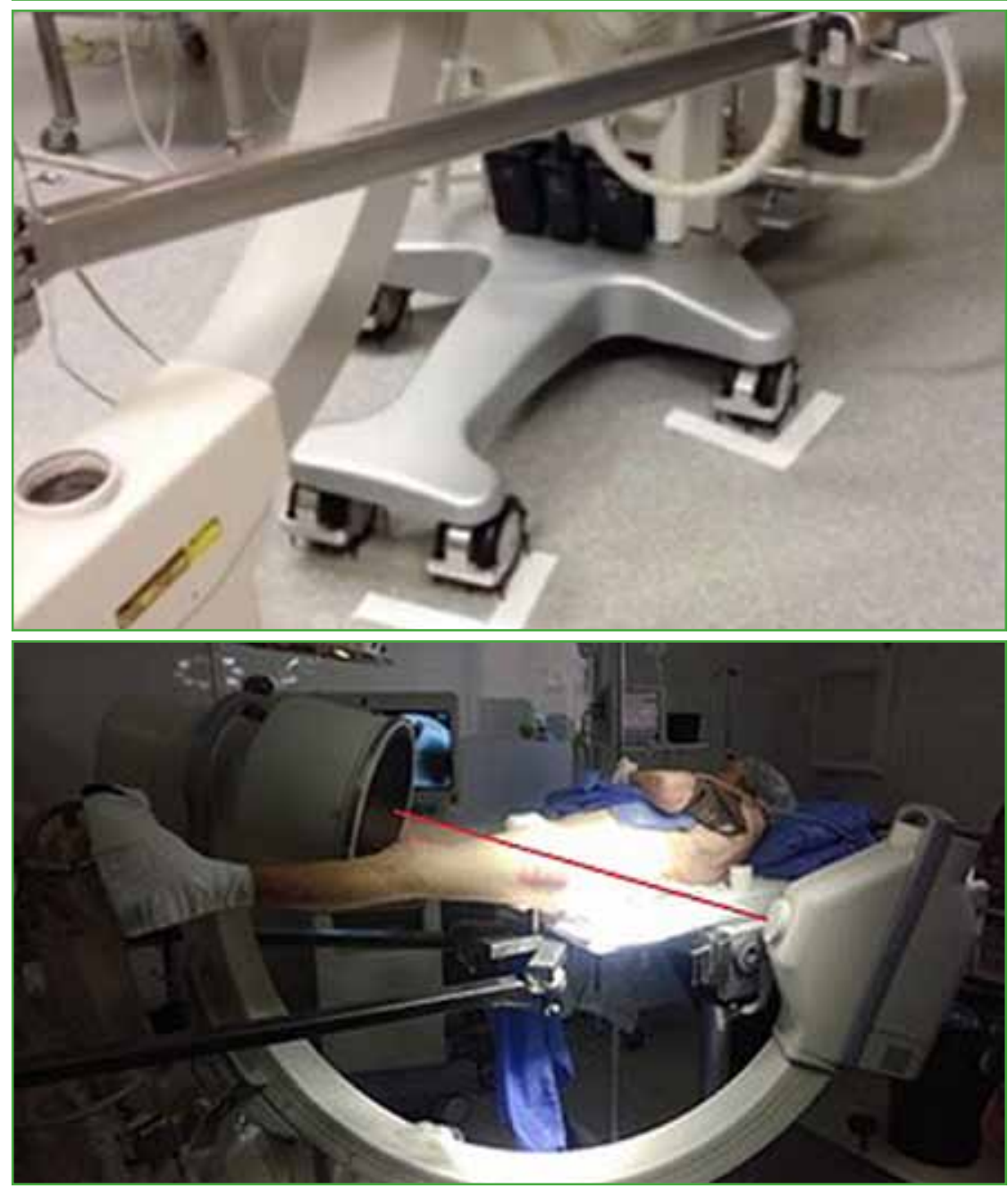

Figura 3. A. Posición del paciente e intensificador de imágenes. B. Marcado en el piso para lograr una adecuada y más rápida posición del intensificador por parte del técnico radiólogo. C. Imagen de la posición del equipo en perfil con ángulo de $20^{\circ}$ para compensar la anteversión del fémur y tener una visualización más clara. 


\subsection{Reducción cerrada}

La reducción debe ser anatómica, así lo indican Krischak y cols. ${ }^{16}$ al analizar la relevancia de la reducción en el resultado final. Trataron a 31 pacientes con reducción cerrada y comunican que las complicaciones no se relacionaron con las reducciones no anatómicas, sobre todo en varo, y que cuando la fractura valguizó, el resultado fue satisfactorio.

La técnica de reducción a cielo cerrado requiere de una mesa ortopédica de tracción y, en general, debe aplicarse al miembro tracción, rotación interna y abducción o aducción, según necesidad, si, con el intento a cielo cerrado, no se obtiene la reducción buscada deberá recurrirse a técnicas de reducción percutáneas o a una reducción abierta.

\subsection{Reducción percutánea}

Hay técnicas percutáneas, aunque no son muy usadas, que mejoran la reducción sin la necesidad de realizar abordajes formales.

Su y cols. ${ }^{17}$ presentan su experiencia con métodos de reducción percutánea en 25 casos de fracturas que fueron irreductibles bajo maniobras externas. El método consiste en colocar dos clavijas de Kirschner percutáneas, cuidando el paquete femoral (por palpación) y, luego con maniobras aplicadas a las clavijas, lograr la reducción y la posterior osteosíntesis con tres tornillos canulados. La tasa de consolidación fue del 100\% y hubo dos casos de necrosis.

En 2019, Yu y cols. ${ }^{18}$ consiguen con una técnica similar muy buenos resultados en 32 pacientes, y hacen especial énfasis en la planificación y el cuidado de las estructuras nobles.

Mahajan y cols. ${ }^{19}$ proponen otro tipo de método percutáneo de reducción que consiste en colocar un alambre de Kirschner que fije la cabeza femoral al acetábulo y, de esta forma, movilizar el fragmento distal para lograr una reducción anatómica. Esta técnica realizada en 15 pacientes logró muy buenos resultados, sin necesidad de la apertura del foco.

\subsection{Reducción abierta}

Básicamente se han descrito y utilizado dos abordajes para una reducción abierta: el anterolateral de WatsonJones y el anterior de Smith-Petersen. Cada uno tiene sus ventajas y desventajas.

El abordaje de Watson-Jones (1936) desarrolla el plano intermuscular existente entre el tensor de la fascia lata y el glúteo medio; una de sus ventajas es que, por la misma incisión, se puede efectuar la reducción de la fractura y también colocar la osteosíntesis, pero como desventaja no permite observar las fracturas que son más subcapitales. ${ }^{20}$

Por otro lado, el abordaje de Smith-Petersen (1917) desarrolla un plano internervioso entre el sartorio (nervio femoral) y el tensor de la fascia lata (nervio glúteo superior), tiene la gran ventaja de abordar la cadera desde anterior, lo que permite no solo observar la fractura adecuadamente, sino también colocar instrumentos para la reducción con comodidad, pero requiere de un segundo abordaje para colocar el implante de fijación. ${ }^{21}$

No existen muchos artículos que determinen qué abordaje es más adecuado para el tratamiento de las fracturas de cuello femoral desplazadas. Slobogean y cols. ${ }^{22}$ llevaron a cabo una encuesta en la cual participaron especialista de toda la sociedad canadiense y la Orthopaedic Trauma Association, preguntaron sobre la elección del abordaje: el 19,9\% eligió el de Watson-Jones y el 13,8\%, el de Smith-Petersen.

Molnar y Routt ${ }^{23}$ publican el primer artículo con el uso del abordaje anterior de Smith-Petersen para el tratamiento de las fracturas de cuello femoral. Utilizando la rama inferior del abordaje tradicional y seccionando el recto anterior describen una excelente visualización del cuello que les permite la reducción mediante diferentes métodos, con muy buena exposición.

Lichstein y cols..$^{24}$ llevaron a cabo un estudio en cadáveres que compara los dos abordajes con el objetivo de demostrar qué abordaje permite más visualización y palpación. Llegaron a la conclusión de que el abordaje de Smith-Petersen, sin tenotomía del recto anterior, expone $2,4 \mathrm{~cm}^{3}$ más de cuello y $1,6 \mathrm{~cm}^{3}$ de superficie articular que el abordaje de Watson-Jones y que, con tenotomía, expone $3,3 \mathrm{~cm}^{3}$ y $1,6 \mathrm{~cm}^{3}$ más, respectivamente. Sin embargo, el abordaje anterior plantea el riesgo de lesión del femorocutáneo hasta en el 60\%; por lo tanto, hacen hincapié en una adecuada disección para, de esta forma, disminuir la incidencia de neuropraxia.

Todavía sigue siendo controvertido cuál es el mejor abordaje y seguramente dependerá de la comodidad del cirujano. Es importante destacar que un traumatólogo que trate este cuadro debe estar familiarizado con las dos opciones. 


\section{Elección del implante}

Luego de la reducción anatómica, se deberá evaluar cuál es el implante que mejor se adapta a la fractura. En la actualidad, existen múltiples opciones de osteosíntesis, pero probablemente y, sobre todo en nuestro medio, la mayor controversia sea entre la colocación de tornillos canulados o clavo compresivo deslizante (CCD).

En un estudio biomecánico, Giannoudis y Panteli ${ }^{25}$ comparan las propiedades de estos dos implantes y concluyen en que, para fracturas desplazadas con trazos más estables (Pawels I y II), tanto los tornillos canulados como el CCD parecerían ser una excelente opción, mientras que, para las fracturas más verticales (Pawels III), el CCD asociado a un tornillo antideslizante aporta más estabilidad.

Bhandari y cols. ${ }^{26}$ llegan a una conclusión similar, prefieren el CCD en los trazos más inestables y pacientes menos colaboradores.

Wu-ren Hou y Min-ou $\mathrm{Xu}^{27}$ realizaron un estudio comparativo entre CCD y tres tornillos canulados en pacientes jóvenes con fracturas de cuello femoral conminutas, y no hallaron diferencias significativas en el tiempo de cirugía, el sangrado y la estancia hospitalaria. Pero sí a favor del CCD más tornillos antirrotatorios en cuanto al tiempo de consolidación (151 vs. 162; p <0,01) y el Harris Hip Score (91 vs. 85; p = 0,01).

En 2019, Cha y cols. ${ }^{28}$ publican una revisión sistemática de la bibliografía sobre esta controversia. Evalúan 18 estudios y concluyen en que la mayoría compara resultados mecánicos y no clínicos, por lo que es imposible determinar qué opción de implante es mejor para cada situación.

En el estudio FAITH (Fracture fixation in the operative management of hip fractures) multicéntrico, internacional, aleatorizado, se evaluaron los riesgos de reoperación luego de una osteosíntesis de cuello femoral, utilizando CCD versus tornillos canulados. El estudio incluyó a 1108 pacientes y no se hallaron diferencias de tasas de reoperación, aunque sugieren el tornillo placa deslizante en fumadores, fracturas desplazadas y basicervicales. ${ }^{29}$

Ma y cols..$^{30}$ publicaron una revisión sistemática sobre el uso de CCD versus tornillos canulados para tratar las fracturas de cuello femoral. No hallaron diferencias en los resultados funcionales (Harris Hip Score) en el grupo de $\mathrm{CCD}$, hubo menos complicaciones y una consolidación más rápida; por ello, concluyen en que su uso parecería ser más eficaz para tratar las fracturas de cuello femoral.

\section{Factores determinantes en la colocación de la osteosíntesis}

Cuando se decide colocar tornillos canulados de 6,5 o $7 \mathrm{~mm}$, existen múltiples factores que condicionarán la eficacia de la técnica: ¿qué cantidad?, ¿en qué posición?, ¿con qué configuración? y ¿en qué secuencia? Si bien no hay un consenso absoluto, sí la coincidencia es amplia en ciertos aspectos técnicos que serían beneficiosos para un mejor resultado final.

\subsection{Punto de entrada}

Los tornillos se deben colocar proximales al nivel del trocánter menor, en la zona esponjosa y no en la cortical del fémur proximal lateral, de manera de disminuir zonas de concentración de estrés y el consiguiente riesgo de fractura subtrocantérica.

Kloen y cols. ${ }^{31}$ reportan cuatro casos en los que, luego de la colocación de tornillos canulados, los pacientes sufrieron una fractura subtrocantérica y concluyen en que la única variable repetida en los cuatro casos es un punto de entrada por debajo del trocánter menor.

Sensoz y cols..$^{32}$ hicieron un análisis finito de los elementos para evaluar las complicaciones relacionadas con un punto de entrada, y mostraron que la colocación de tornillos por debajo del trocánter menor aumenta significativamente las posibilidades de generar una fractura subtrocantérica.

\subsection{Cantidad de tornillos}

La cantidad de tornillos por colocar tiene una directa relación con el tipo de fractura que se tratará. En 1987, Swiontkowski y cols. ${ }^{33}$ realizaron un estudio en hueso cadavérico comparando la estabilidad con tres, cuatro y cinco tornillos canulados, sometiéndolos no solo a compresión axial, sino también a fuerzas de torsión y flexión. Los autores demostraron que no existe ningún beneficio mecánico en colocar más de tres tornillos y llaman la atención sobre la importancia de evaluar la calidad ósea.

Sin embargo, varios estudios comunicaron mayor estabilidad en el montaje cuando hay tres tornillos. En 2003, Maurer y cols. ${ }^{34}$ compararon la estabilidad entre dos y tres tornillos, y demostraron que el tercer tornillo aumenta la resistencia a la compresión axial. 
En 2006, Krastman y cols. ${ }^{35}$ evaluaron 112 casos de fracturas de cuello femoral tratados con dos tornillos canulados y demostraron que esta cantidad es adecuada en fracturas no desplazadas, la tasa de consolidación fue del $100 \%$ y no hubo complicaciones.

En todas las fracturas desplazadas de nuestra serie, intentamos colocar, al menos, tres tornillos, mientras que, en las fracturas no desplazadas o impactadas en valgo, la colocación de dos tornillos resultó suficiente.

\subsection{Arandelas}

Otro tema controvertido es el uso de arandelas. En 2005, Zlowodzki y cols. ${ }^{36}$ evaluaron múltiples factores predictivos de falla de la osteosíntesis en fracturas de cuello femoral, y la ausencia de arandelas fue un factor estadísticamente significativo $(\mathrm{p}=0,03)$ de falla $\mathrm{y}$, por lo tanto, aconsejan su uso.

Casi una década después, el mismo autor ${ }^{37}$ realiza un estudio biomecánico comparando el máximo torque posible colocando tornillos con arandela o sin ella, en hueso osteoporótico. Hubo una diferencia estadísticamente significativa $(\mathrm{p}<0,01)$ a favor del uso de arandelas como método que permite más compresión y torque al colocar el tornillo.

Sin embargo, en la práctica diaria, muchas veces, la colocación de todas las arandelas es imposible, ya que los tornillos vecinos no lo permiten, respecto a esto no hay mucho publicado; por lo tanto, sería adecuado colocar solo arandelas que no dificulten la colocación de los demás tornillos y, de esa forma, lograr la construcción más estable.

\subsection{Configuración}

En 2004, Selvan y cols. ${ }^{38}$ compararon montajes diferentes de tornillos, tres con configuración triangular y tres con configuración lineal en un estudio biomecánico. Sometiéndolas a pruebas de desplazamiento, carga máxima tolerada y energía absorbida, demostraron claramente que las configuraciones triangulares soportan una carga máxima más alta, menos desplazamiento y más absorción de energía antes del fallo.

Recientemente Guo y cols. ${ }^{39}$ intentan definir el montaje ideal de tornillos evaluando una serie de 96 pacientes con fractura de cuello femoral tratados con osteosíntesis. Concluyen en que la configuración triangular con vértice inferior es la que logra mejores resultados y resaltan la importancia de colocar el tornillo posterior y superior más central para, de esta forma, evitar la "violación de la cortical" posterior del cuello.

En 2014, Gümüta y cols. ${ }^{40}$ presentan un trabajo biomecánico donde comparan diferentes métodos de osteosíntesis para el tratamiento de las fracturas más inestables o verticales, y comunican que, al agregar un tornillo transversal al calcar, aumenta significativamente la estabilidad del montaje.

Con la misma intención de aumentar la estabilidad en los escenarios más desfavorables, Collinge y $\mathrm{Mir}^{41}$ proponen agregar a la fijación de los tornillos una placa tercio de tubo en la cara medial del cuello a modo de sostén para así disminuir la posibilidad de colapso en varo.

En 2017, Ye y cols. ${ }^{42}$ publican su experiencia con esta técnica en 28 pacientes $<60$ años con fractura medial de cadera Pawels III. La tasa de consolidación fue del $89 \%$ y no hubo casos de necrosis. Los autores llegan a la conclusión de que es un recurso adecuado y que no afecta la vascularización de la cabeza femoral.

En 2019, Hoffman y cols..$^{43}$ publican su estudio cadavérico donde llaman la atención respecto a la colocación del tornillo posterior en un montaje triangular de vértice inferior. Evaluaron 10 especímenes y demostraron, por disección y tomografía, que siete de los 10 violaban o perforaban la cortical posterior; por esta razón, pregonan la colocación de este tornillo más central.

Se dispone de poca bibliografía sobre la distancia entre los tornillos. Gurusamy y cols. ${ }^{44}$ evaluaron 395 fracturas mediales de cadera, y comunicaron que la distancia entre los tornillos se relaciona con la posibilidad de seudoartrosis y que cuanto mayor distancia hay entre los tornillos en el perfil, más posibilidades hay de lograr la consolidación.

Parecería entonces que existe suficiente evidencia para la adopción de un montaje triangular, de vértice inferior y con la mayor distancia posible entre los tornillos que no haga correr riesgos de violación de las corticales, especialmente con el tornillo proximal y posterior. Si se trata de un trazo vertical, cuando el riesgo de colapso en varo aumenta, la colocación de un tornillo perpendicular al calcar o de una placa de sostén mediales parece un recurso válido que se podrá utilizar según el criterio del cirujano.

Por último, es importante destacar que existe una secuencia de colocación de tornillos que nos asegura la menor posibilidad de pérdida de la reducción durante su colocación; primero se debe colocar el tornillo inferior central para, de esta forma, poder contrarrestar las fuerzas de colapso en varo, pero sin darle compresión definitiva, luego se coloca el tornillo posterior, lo que disminuye las posibilidades de caída posterior y finalmente el superior y el anterior. Una vez colocados los tres tornillos, se deberá dar compresión primero a los superiores con el fin de evitar la desviación en varo que podría ocurrir si le damos compresión primero al tornillo inferior (Figura 4). 

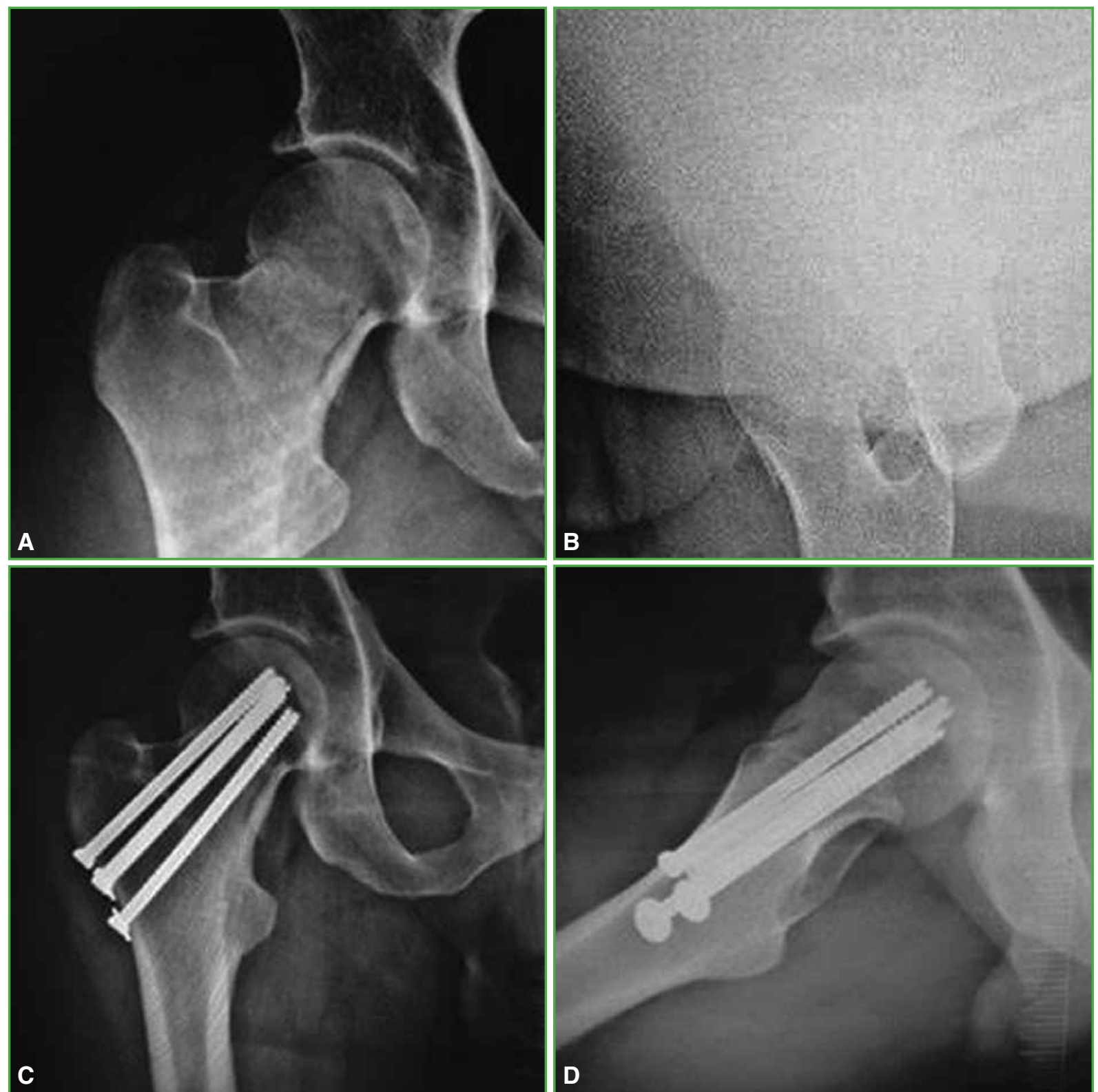

Figura 4. A. Radiografía de una fractura de cuello femoral, de frente. B. Radiografía de la fractura, de perfil quirúrgico.

C. Radiografía posoperatoria de frente. Se observa la correcta colocación de cuatro tornillos en forma romboidal.

D. Radiografía posoperatoria de perfil. Se observa la correcta colocación de los tornillos.

\section{Capsulotomía}

Existen diferentes opiniones acerca de qué conducta se debe adoptar en cuanto a realizar una capsulotomía o no para descomprimir el hematoma en las fracturas de cuello femoral desplazadas en el adulto joven.

Varios estudios demuestran beneficios con este gesto, Ly y Swiontkowski ${ }^{45}$ evaluaron el rol de la capsulotomía dentro del tratamiento de las fracturas de cuello femoral, y concluyen en que la controversia sigue, pero hasta que se disponga de información adecuada contraria (estudios prospectivos con grupo de control) recomiendan su realización. 
Sin embargo, Triplet y cols. ${ }^{46}$ evaluaron a 62 pacientes jóvenes, 32 de ellos fueron sometidos a una capsulotomía. Del total de pacientes, siete desarrollaron necrosis, a cinco de ellos se les había realizado una capsulotomía. Por lo tanto, llegaron a la conclusión de que la capsulotomía no previene el desarrollo de necrosis.

En nuestra serie sin punciones ni capsulotomías, salvo en las reducciones abiertas, registramos una incidencia habitual de necrosis por lo que no realizamos ningún gesto además de la cirugía propiamente dicha, ya sea a cielo abierto o cerrado.

\section{Manejo posoperatorio}

Se discute poco sobre cuál es el protocolo posoperatorio más adecuado. Está claro que nos enfrentamos a dos tipos diferentes de pacientes, por un lado, el paciente joven que puede no cargar peso y eso no afectará su vida diaria, pero, por el otro, los pacientes más añosos a quienes la restricción de la carga afectará mucho más su actividad cotidiana.

Florschutz y cols. ${ }^{14}$ recomiendan la carga inmediata, según tolerancia, con andador para los pacientes con fracturas no desplazadas o impactadas en valgo; sin embargo, difieren la carga hasta las ocho semanas si las fracturas son desplazadas y sobre todo si la conminución es posterior.

Conflicto de intereses: Los autores no declaran conflictos de intereses.

ORCID de S. Pereira: https://orcid.org/0000-0001-9475-3158

ORCID de G. Vindver: https://orcid.org/0000-0003-3858-6687

\section{BIBLIOGRAFÍA}

1. Manninger G, Kazar G, Fekete K, Fekete S, Frenyo F. Significance of urgent (within 6 h) internal fixation in the management of fractures of the neck of the femur. Injury 1989;20(2):101-5. https://doi.org/10.1016/0020-1383(89)90152-6

2. Loizou M, Parker J. Avascular necrosis after internal fixation of intracapsular hip fractures; a study of the outcome for 1023 patients. Injury 2009;40(11):1143-6. https://doi.org/10.1016/j.injury.2008.11.003

3. Upadhyay A, Jain P, Mishra P, Maini L, Gautum VK. Delayed internal fixation of fractures of the neck of the femur in young adults. A prospective, randomised study comparing closed and open reduction. J Bone Joint Surg Br 2004;86(7):1035-40. https://doi.org/10.1302/0301-620x.86b7.15047

4. Papakostidis C, Panagiotopoulos A, Piccioli A, Giannoudis P. Timing of internal fixation of femoral neck fractures. A systematic review and meta-analysis of the final outcome. Injury 2015;46(3):459-66. https://doi.org/10.1016/j.injury.2014.12.025

5. Pereira S, Calvo X, Lugones A, Vindver G, Bidolegui F. Osteosíntesis con tornillos canulados en fracturas mediales de cadera Factores pronósticos y resultados en 93 casos Rev Asoc Argent Ortop Traumatol 2014;79(1):27-34. https://www.aaot.org.ar/revista/2014/n1/Rev_Asoc_Argent_Ortop_Traumatol_2014_79_(1)_27-34_Pereira.pdf

6. Egol K, Koval KJ, Oh CK. Does a traction-internal rotation radiograph help to better evaluate fractures of the proximal femur? Bull NYU Hosp Jt Dis 2008;66(2):102-6. PMID: 18537778

7. Temmesfeld M, Dolatowski FC, Borthne A, Utvag SE, Hoelsbrekken SE. Cross-table lateral radiographs accurately predict displacement in valgus-impacted femoral neck fractures. J Bone Joint Surg 2019:e0037. https://doi.org/10.2106/JBJS.OA.18.00037

8. Frandsen P, Andersen P, Christoffersen H, Per B. Thomsen osteosynthesis of femoral neck fracture: The slidingscrew-plate with or without compression. Acta Orthop Scand_1984;55(6):620-3. https://doi.org/10.3109/17453678408992408

9. Huang TW, Hsu WH, Peng KT, Lee CY. Effect of integrity of the posterior cortex in displaced femoral neck fractures on outcome after surgical fixation in young adults. Injury 2011;42(2):217-22.

https://doi.org/10.1016/j.injury.2010.10.005 
10. Rawall S, Bali K, Upendra B, Garg B, Yadav CS, Jayaswal A. Displaced femoral neck fractures in the young: significance of posterior comminution and raised intracapsular pressure. Arch Orthop Trauma Surg 2012;132:73-9. https://doi.org/10.1007/s00402-011-1395-1

11. Rikli D, Goldhahn S, Blauth M, Mehta S, Cunningham M. PIP Study group. Optimizing intraoperative imaging during proximal femoral fracture fixation - A performance improvement program for surgeons. Injury 2018;49(2):339-44. https://doi.org/10.1016/j.injury.2017.11.024

12. Poyanli OS, Soylemez S, Ozkut AT, Uygur E, Kemah B. Precise placement of lag screws in operative treatment of trochanteric femoral fractures with a new guide system Injury 2015;46(11):2190-95. https://doi.org/10.1016/j.injury.2015.06.003

13. Yang J, Lin L, Chao K, Chuang S, Wu C. Risk factors for nonunion in patients with intracapsular femoral neck fractures treated with three cannulated screws placed in either a triangle or an inverted triangle configuration. J Bone Joint Surg Am 2013;95(1):61-9. https://doi.org/10.2106/JBJS.K.01081

14. Florschutz A, Langford J, Haidukewych G, Koval K. Femoral neck fractures: current management. J Orthop Trauma 2015;29(3):121-9. https://doi.org/10.1097/BOT.0000000000000291

15. Chua D, Jaglal SB, Schatzker J. Predictors of early failure of fixation in the treatment of displaced subcapital hip fractures. J Orthop Trauma 1998;12(4):230-4. https://doi.org/10.1097/00005131-199805000-00002

16. Krischak G, Beck A, Wachter N, Jakob R, Kinzl L, Suger G. Relevance of primary reduction for the clinical outcome of femoral neck fractures treated with cancellous screws. Arch Orthop Trauma Surg 2003;123(8):4049. https://doi.org/10.1007/s00402-003-0571-3

17. Su Y, Chen W, Zhang Q, Li B, Guo M. An irreducible variant of femoral neck fracture: A minimally traumatic reduction technique. Injury 2011;42(2):140-5. https://doi.org/10.1016/j.injury.2010.05.008

18. Yu S, Xu X, Pandey N, Zhao Y, Jing J. A safe percutaneous technique for the reduction of irreducible femoral neck fractures using ultrasound localization of the femoral vascular and nervous structures at the hip. Medicine (Baltimore) 2019;98(15): e15163. https://doi.org/10.1097/MD.0000000000015163

19. Mahajan RH, Kumar S, Mishra B. Technique for gentle accurate reproducible closed reduction of intracapsular fracture of neck of femur. Injury 2017;48(3):789-790. https://doi.org/10.1016/j.injury.2016.10.030

20. Watson-Jones R. Fractures of the neck of the femur. Br J Surg 1936;23(92):787-808. https://doi.org/10.1002/bjs.1800239213

21. Smith-Petersen MN. A new supra-articular subperiosteal approach to the hip joint. J Bone Joint Surg Am 1917;s215(8):592.

22. Slobogean GP, Sprague SA, Scott T, McKee M, Bhandari M. Management of young femoral neck fractures: is there a consensus? Injury 2015;46(3):435-40. https://doi.org/10.1016/j.injury.2014.11.028

23. Molnar RB, Routt ML. Open reduction of intracapsular hip fractures using a modified Smith-Petersen surgical exposure. J Orthop Trauma 2007;21(7):490-4. https://doi.org/10.1097/BOT.0b013e31804a7f7f

24. Lichstein P, Kleimeyer J, Githens M, Vorhies J, Gardner M, Bellino M, et al. Does the Watson-Jones or modified Smith-Petersen approach provide superior exposure for femoral neck fracture fixation? Clin Orthop Relat Res 2018;476(7):1468-76. https://doi.org/ 10.1097/01.blo.0000533627.07650.bb

25. Giannoudis P, Panteli M. Biomechanical rationale for implant choices in femoral neck fracture fixation in the nonelderly. Injury 2015;46(3):445-52. https://doi.org/10.1016/j.injury.2014.12.031

26. Bhandari M, Tornetta P 3rd, Hanson B, Swiontkowski M. Optimal internal fixation for femoral neck fractures: multiple screws or sliding hip screws? J Orthop Trauma 2009;23(6):403-7. https://doi.org/10.1097/BOT.0b013e318176191f

27. Wu-ren Hou, Min-ou Xu. [Comparison among three cannulated screws and dynamic hip screw combined with antirotation screw for comminuted fractures of femoral neck]. China J Orthop Traumatol 2015;28(9):796-801. [En chino]. PMID: 26647559

28. Cha Y, Yoo J, Wang S, Kim K, Choy W, Hwang S. Biomechanical evaluation of internal fixation of Pauwels type III femoral neck fractures: A systematic review of various fixation methods. Clin Orthop Surg 2019;11(1):1-14. https://doi.org/10.4055/cios.2019.11.1.1

29. Fixation using Alternative Implants for the Treatment of Hip fractures (FAITH) Investigators. Fracture fixation in the operative management of hip fractures (FAITH): an international, multicentre, randomised controlled trial. Lancet 2017;389(10078):1519-27. https://doi.org/10.1016/S0140-6736(17)30066-1

30. Ma J, Kuang M, Xing F, Zhao Y, Chen H, Zhang L. Sliding hip screw versus cannulated cancellous screws for fixation of femoral neck fracture in adults: A systematic review. Int J Surg 2018;52:89-97.

https://doi.org/10.1016/j.ijsu.2018.01.050 
31. Kloen P, Rubel I, Lyden J, Helfet D. Subtrochanteric fracture after cannulated screw fixation of femoral neck fractures: A report of four cases. J Orthop Trauma 2003;17(3):225-9. https://doi.org/10.1097/00005131-200303000-00013

32. Sensoz E, Mehmet F, Acar V. Finite element analysis of the impact of screw insertion distal to the trochanter minor on the risk of iatrogenic subtrochanteric fracture. Proc Inst Mech Eng H 2018;232(8):807-18. https://doi.org/10.1177/0954411918789963

33. Swiontkowski MF, Harrington RM, Keller TS. Torsion and bending analysis of internal fixation techniques for femoral neck fracture: the roll of implant design and bone density. J Orthop Res 1987;5:433-44. https://doi.org/10.1002/jor.1100050316

34. Maurer S, Wright K, Koval K, Zuckerman F. Two or three screws for fixation of femoral neck fractures? Am J Orthop (Belle Mead NJ) 2003;32(9):438-42. PMID: 14560825

35. Krastman P, van den Bent RP, Krijnen P, Schipper IB. Two cannulated hip screws for femoral neck fractures: treatment of choice or asking for trouble? Arch Orthop Trauma Surg 2006;126(5):297-303. https://doi.org/10.1007/s00402-006-0143-4

36. Zlowodzki M, Weening B, Petrisor B, Bhandari M. The value of washers in cannulated screw fixation of femoral neck fractures. Trauma 2005;59(4):969-75. https://doi.org/10.1097/01.ta.0000188130.99626.8c

37. Zlowodzki M, Wijdicks C, Armitage B, Cole A. Value of washers in internal fixation of femoral neck fractures with cancellous screws: a biomechanical evaluation. J Orthop Trauma 2015;29(2):e69-72. https://doi.org/10.1097/BOT.0000000000000172

38. Selvan VT, Oakley J, Rangan A, Al-Lami MK. Optimum configuration of cannulated hip screw for the fixation of intracapsular hip fracture: a biomechanical study. Injury 2004;35(2):136-41. https://doi.org/10.1016/S0020-1383(03)00059-7

39. Guo I, Dong W, Qin S, Zhang Y. Definition of ideal configuration for femoral neck screw fixation in older people. Sci Rep 2019;9:12895. https://doi.org/10.1038/s41598-019-48258-2

40. Gümüştaş SA, Tosun HB, Ağır İ, Orak MM, Onay T, Okçu G. Influence of number and orientation of screws on stability in the internal fixation of unstable femoral neck fractures. Acta Orthop Traumatol Turc 2014;48(6):673-8. https://doi.org/10.3944/AOTT.2014.14.0088

41. Collinge $\mathrm{C}$, Mir H. Application of a medial buttress plate may prevent many treatment failures seen after fixation of vertical femoral neck fractures in young adults. Med Hyp 2015;84(5):429-33. https://doi.org/10.1016/j.mehy.2015.01.029

42. Ye Y, Chen K, Tian K, Li W, Mauffrey C, Hak DJ. Medial buttress plate augmentation of cannulated screw fixation in vertical unstable femoral neck fractures: surgical technique and preliminary results. Injury 2017;48(10):2189-93. https://doi.org/10.1016/j.injury.2017.08.017

43. Hoffman J, Kellam J, Routt C, Gary J. Is the cranial and posterior screw of the "inverted triangle" configuration for femoral neck fractures safe? J Orthop Trauma 2019;33(7):331-4. https://doi.org/10.1097/BOT.0000000000001461

44. Gurusamy K, Parker MJ, Rowlands TK. The complication of displaced intracapsular fractures of the hip. The effect of screw positioning and angulation on fracture healing. J Bone Joint Surg Br 2005;87:632-4. https://doi.org/10.1302/0301-620X.87B5.15237

45. Ly TV, Swiontkowski MF. Treatment of femoral neck fractures in young adults. Instr Course Lect 2009;58:69-81. PMID: 19385521

46. Triplet JJ, Sullivan B, Taylor BC. The influence of capsulotomy for intracapsular hip fractures. J Long Term Eff Med Implants 2018;28(3):187-92. https://doi.org/10.1615/JLongTermEffMedImplants.2018028556 